\title{
Concentration Modulation Effect on Weakly Nonlinear Thermal Instability in a Rotating Porous Medium
}

\author{
P. Kiran \\ Department of Mathematics, Chaitanya Bharathi Institute of Technology, Gandipet, Hyderabad, \\ Telangana-500075, India. \\ $\dagger$ Corresponding Author Email: pallekiran_maths@cbit.ac.in
}

(Received July 13, 2019; accepted January 14, 2020)

\begin{abstract}
The present article is to study mass transfer in a rotating porous layer subjected to imposed time-periodic solutal boundaries. A weakly nonlinear analysis is applied to investigate mass transfer in a porous medium. The mass transfer coefficient is calculated by cubic Ginzburg Landau (GLE) amplitude equation. In this article the stationary convection is discussed in the presence of rotating solutal Rayleigh number. The amplitude equation (GLE) is solved numerically to calculate finite temporal convective amplitude. This amplitude is used to find Sherwood number in terms of the various system parameters. The effect of individual parameters on mass transport is discussed in detail in the presence of lower rotational rates. The onset of convection is discussed through the stability curves for stationary and oscillatory solutal critical Rayleigh number as a function of wavenumber. Further, it is found that the mass transfer enhances for modulated system than un-modulated system. Internal solutal number $\mathrm{Si}$ is to enhances for higher values and diminishes the mass transfer for lower values. Finally, it is also found that rotation and solutal modulation can be effectively used to enhance or diminish the mass transfer.
\end{abstract}

Keywords: Darcy convection; Concentration modulation; Rotation; Nonlinear theory.

\section{NOMENCLATURE}

amplitude of convection amplitude of modulation depth of the porous medium velocity vector $(\mathrm{u}, \mathrm{v}, \mathrm{w})$ acceleration due to gravity Critical wave number Sherwood number Solute Vadász number $\operatorname{Vas}=\frac{\phi v d^{2}}{K_{s} \kappa_{s}}$ reduced pressure Internal solute number $\mathrm{S}_{\mathrm{i}}=\frac{\mathrm{Qd}^{2}}{\mathrm{\kappa}_{\mathrm{s}}}$ Solutal Rayleigh number $\mathrm{Ra}_{\mathrm{s}}=\frac{\alpha_{\mathrm{s}} \mathrm{gv} \Delta \mathrm{SKd}}{v \kappa_{\mathrm{s}}}$ Critical Rayleigh number taylor number $\mathrm{Ta}=\left(\frac{2 \Omega_{0} \mathrm{~d}^{2}}{v}\right)^{2}$ solute solute difference across the porous medium time horizontal and vertical co-ordinates coefficient of solute expansion $\beta^{2} \quad$ Horizontal wave number $\mathrm{k}_{\mathrm{c}}^{2}+\pi^{2}$

$\chi \quad$ perturbation parameter

$\kappa_{s} \quad$ effective solute diffusivity

$\Omega \quad$ frequency of modulation

$\mu \quad$ dynamic viscosity of the fluid

$v \quad$ kinematic viscosity $\left(\frac{\mu}{\rho_{0}}\right)$

$\rho \quad$ fluid density

$\psi \quad$ stream function

$\tau \quad$ slow time $\tau=\chi^{2} \mathrm{t}$

\section{Other symbols}

$\nabla_{1}^{2} \quad \frac{\partial^{2}}{\partial \mathrm{x}^{2}}+\frac{\partial^{2}}{\partial \mathrm{y}^{2}}$

$\nabla^{2} \quad \nabla_{1}^{2}+\frac{\partial^{2}}{\partial \mathrm{z}^{2}}$

Subscripts

$B$ basic state

c critical

0 reference value

Superscripts

, perturbed quantity

* dimensionless quantity 


\section{INTRODUCTION}

The Rayleigh Bénard convection related to a porous media is known as Bénard-Darcy convection. This convection is related to either by buoyancy or by external flow. The convection related to buoyancy is free or natural convection and by external force it is forced convection. The convection is also a major mode of mass transfer in fluids or porous media. Convective mass transfer takes place by the diffusion of random Brownia motion of individual particles in the fluid. Mass transfer is the net movement of mass from one location to another location in the form of stream, phase, fraction or component. There are many situations in general mass transfer occurs, such as evaporation, drying, absorption, precipitation, membrane filtration, and distillation. The study of mass transport is important in many engineering applications involving diffusive and convective transport of chemical species within physical systems. Other applications to mass transfer are the purification of blood in the kidneys and liver, and the distillation of alcohol. Mass transfer includes separation of chemical components in distillation columns, absorbers such as activated carbon beds and absorbers such as scrubbers or stripping. Some other related to porous media is given by Gupta et al. (2017). Related to porous material such as solidification of binary mixtures, electro-chemistry, geophysical systems, migration of solutes in watersaturated soils, and the migration of moisture through air contained in fibrous insulation.

Thus looking into the above paragraph it is understood that in many practical problems the concentration gradient (see Mohammed, 2013) is a function of both space and time. Hence study of concentration modulation is very important to control convective mass transfer in a porous medium. Also the study of rotating porous media is attracting from researchers due to its challenging theoretical applications in the fields of science and engineering. Some of them are chemical process industry, food process industry and rotating turbo machinery. Studies related convection in porous media was given by Horton and Rogers (1945) and Lapwood (1948). They have extensively investigated the onset of thermal convection in a horizontal porous media saturated with Newtonian fluid. In recent the study of convection and the study of heat mass transfer in a rotating media is given by many authors. Some of them are discussed here: The effect of thermal modulation and rotation on the onset of stationary convection in porous layer is given by Malashetty et al. (2007) subject to the principle of exchange of stabilities. They found that the convection is advanced by the low frequency inphase and lower-wall temperature modulation, and delayed by the out-of-phase modulation. Moli et al. (2014) investigated heat mass transfer analysis in porous media of maxwell fluids. In this paper both linear and nonlinear thermal convection is investigated without modulation. They have discussed various system parameters effect on heat mass transfer.

The effect of rotation speed modulation on heat transfer in a porous layer or fluid layer is given by Bhadauria et al. (2014) and Kiran et al. (2017).

The Ginzburg Landau equation is employed in both papers to derive finite amplitude. It is found that rotational speed modulation is used to control heat transfer in the media. The modulated centrifugal convection in a rotating vertical porous layer distant from the axis of rotation is given by Om et al. (2009). Study of rotation on Brinkman-Lapwood convection with modulation is investigated by Om et al. (2011). They found that by applying the modulation frequency to the rotation speed, it is possible to delay or advance the onset of centrifugal convection. Kiran et al. (2016c,d, 2017) investigated the nonlinear throughflow effects on thermally modulated rotating porous media. It is found that rotation has stabilizing effect on porous media in the presence of modulation. They also found that nonlinear throughflow has dual nature on the onset convection. It is concluded that flow direction may have both stabilizing and destabilizing effect on the system. The effect of throughflow and electric field on the outset of convective instability in a horizontal porous layer saturated by a dielectric nanofluid is investigated by Yadav (2018). Using the Galerkin weighted residuals method the linear theory was employed and discussed stability criteria. Using the complex Ginzburg Landau model the study of rotating fluid convection is given by Kiran et al. (2016a,b). In this paper the temporal convective amplitude is derived using the solvability condition up to the third order. For very low Prandtl number values $\operatorname{Pr}<1$ the study of heat transfer is discussed along with exchange of stabilities. It is found that better results are obtained for an oscillatory convection than stationary convection. Later the extent of research information in the area of convection in porous media is given by Nield and Bejan (2006), Ingham and Pop (2005) and Vafai (2005).

The research on modulated flows are very important due to stabilize or destabilize nature of convective flow. This modulation is gives system performance, system usage and the life span of the system. Instability and transport phenomenon is controlled by modulation only even though there are other physical constraints. There are two types of modulations like thermal and gravity which are investigated by so many authors. Venezian (1969) was the first who performed a linear stability analysis of Rayleigh Bénard convection in a fluid layer under temperature modulation and obtained the correction in the critical Rayleigh number. It was found that onset convection is effectively controlled by modulation. Recently, the research corresponding to 
thermal modulation is given by Kiran et al. (2018a,b) and other references therein. Here they have investigated the effect of modulation on chaotic convection in porous media. The effect of gravity modulation on Rayleigh- Bénard convection is given by Gresho and Sani (1970). The effect of gravity modulation and throughflow in porous media is given by Kiran (2016c). It is found that nonlinear throughflow having both effects enhancing and reducing heat transfer. The study of weak nonlinear double diffusive magneto convection in a Newtonian liquid under gravity modulation is investigated by Bhadauria et al. (2015). The finite amplitude is found to enhance for Prandtl and magnetic Prandtl number, Levies number, and amplitude of modulation. The effect of gravity modulation on internally heated porous media is studied by Srivastava et al. (2018). Using the stationary Landau equation they found that heat mass transfer can be controlled by both concepts g-jitter and internal heat source. An oscillatory mode of convection in a rotating fluid layer under gravity modulation is given by Kiran et al. (2018c, 2020) and the corresponding updated literature of gravity modulation therein. The study of convection in a rotating porous media is given by Pearlstein (1981), Patil et al. (1990), Desaive et al. (2002), Suthar et al. (2011), Bhadauria et al. (2012), Kiran et al. (2017) can be named as few.

Studies related to temperature and gravity modulation given are by Kiran et al. (2017c), Kiran et. al. (2016c,d; 2017a,b; 2018), Umavathi (2013), Bhadauria et al. (2013a, 2014a-d) and Srivastava et al. (2013). The effect of gravity modulation on Rayleigh Benard convection for an oscillatory mode of convection is given by Kiran et al. (2020). The complex Ginzburg Landau equation is derived to quantify finite amplitude and heat transfer analysis there in. The study of heat mass transfer for stationary and oscillatory convection in an OldroydB fluid layer is given by Raghunatha et al. (2019). They show that the effect of Pr is to decrease the heat and mass transfer in the case of oscillatory motions. They also found that upon increasing relaxation and retardation parameters is to decrease and increase heat and mass transfer for stationary case, while an opposing behavior for oscillatory motions. The study of heat and mass transfer between two concentric vertical cylinders of infinite length is given by Singh and Singh (2019). Various system parameters on velocity profiles and on heat mass transfer is discussed and evaluated the skin friction coefficient. Also studies on concentration modulation in a porous media not even a single paper is found. Till today studies related thermal, gravity, magneto and rotation modulation is observed but not concentration modulation in porous media.

Recently the studies of Rayleigh and Darcy Benard convections under concentration modulation are investigated by Gupta et al. (2017), Gupta (2018) and Prakash et al. (2018). An analytical study of weakly nonlinear mass transfer in rotating fluid layer under time-periodic concentration/gravity modulation is given by Gupta et al. (2017). They found that the effect of increasing Taylor number is to de-crease the mass transfer and concluded that the mass transport can be controlled by suitably adjusting the frequency and amplitude of the modulation. Study of mass transport in rotating couple stress fluid layer under concentration modulation is given by Gupta (2018). Using the Ginzburg-landau equation the rate of mass transfer is quantified in terms of the system parameters. It is found that, the effect of Taylor number is to stabilize the system and decrease the mass transfer. But the value of Sh increases upon increasing the values of Schmidt number and Couple stress parameter. Prakash et al. (2018) is investigated the magentosolutal convection in Newtonian fluid layer under the effects of concentration modulation. While performing the weakly nonlinear theory they have obtained the mass transfer in terms of Sherwood number.

To the best of author's knowledge, there is no nonlinear study available in the literature in which the effect of concentration modulation has been considered in a modulated porous layer with internal solute. This motivated to perform a nonlinear stability analysis and study the combined effect of internal solute and concentration modulation in a porous medium.

\section{MATHEMATICAL MODEL}

Darcy convection in a rotating porous media is considered. The problem is extended in $\mathrm{x}$ direction infinitely and confined between two parallel horizontal planes at $\mathrm{z}=0$ and $\mathrm{z}=\mathrm{d}$. This porous layer is internally soluted (given in Fig.1) and modulated. Further the Boussinesq approximation is employed in which the density variations are consider along with acceleration due to gravity. A cartesian frame of reference is chosen in which origin lies on the lower plate and the z-axis is vertically upward. The solute is heavier at upper plate than the lower plate and $\Delta \mathrm{S}$ is the solutal difference between the plates. Under these assumptions the governing mathematical equations are given by (Bhadauria et al. 2013a):

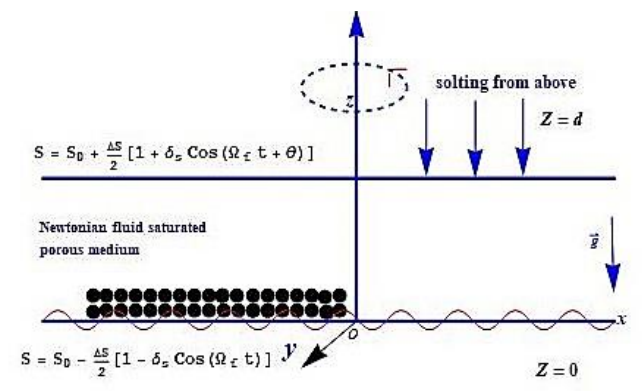

Fig. 1. The Physical Configuraion of the problem. 
$\nabla \cdot \vec{q}=0$

$\frac{1}{\varphi} \frac{\partial \vec{q}}{\partial t}+2(\vec{\Omega} \times \vec{q})=-\frac{1}{\rho_{0}} \nabla p+\frac{\rho}{\rho_{0}} g-\frac{\mu(s)}{\rho_{0} K} \vec{q}$,

$\frac{\partial S}{\partial t}+(\vec{q} . \nabla) S=\kappa_{s} \nabla^{2} S+Q\left(S-S_{0}\right)$,

$\rho=\rho_{0}\left[1-\alpha_{s}\left(S-S_{0}\right)\right]$,

The externally imposed solutal boundary conditions are consider in the following two ways, at first the concentration is more at upper plate and less at lower plate (given by Prakash et al. (2018) and Gupta 2018):

$$
\begin{aligned}
S & =S_{0}-\frac{\Delta S}{2}\left[1-\chi^{2} \delta_{s} \cos (\Omega t)\right] \text { at } \mathrm{z}=0 \\
& =S_{0}+\frac{\Delta S}{2}\left[1+\chi^{2} \delta_{s} \cos (\Omega t+\theta)\right] \text { at } \mathrm{z}=\mathrm{d}
\end{aligned}
$$

where $\delta_{\mathrm{s}}$ is the amplitude of modulation, $\Delta \mathrm{S}$ is the solutal difference across the planes, $\Omega_{f}$ is the modulation frequency, $\theta$ is the phase difference and $\chi$ indicates the smallness of solute modulation. The constants and variables used in the above Eqs. (1)(5) have their usual meanings and are given in the nomenclature. The term $(\vec{\Omega} \times \vec{q})$ (in Eq. (2)) represents rotation of the layer and $Q\left(S-S_{0}\right)$ (in Eq. (3)) represents internal solutal term. The porous layer is rotating about its vertical access uniformly.

\subsection{Conduction State}

In this state the transfer of mass is in touch with plate only. Where the internal mass transfer is due to microscopic collisions of particles and their movement within a body. At this state the physical variables are considered as $\vec{q}_{b}=(0,0,0)$. The other quantities of the basic state are:

$\rho=\rho_{b}(z), p=\mathrm{p}_{b}(z)$, and $S=\mathrm{S}_{b}(z, t)$.

Substituting Eq. (6) into Eqs. (1)-(4), we get the following relations which helps to define the basic state pressure and concentration:

$$
\begin{aligned}
& \frac{\mathrm{dp}_{b}}{d z}=-\rho_{b} g, \\
& \kappa_{s} \frac{d^{2}\left(S_{b}-S_{0}\right)}{d z^{2}}+Q\left(S_{b}-S_{0}\right)=\frac{\partial S_{b}}{\partial t} \\
& \rho=\rho_{0}\left[1-\alpha_{s}\left(S_{b}-S_{0}\right)\right]
\end{aligned}
$$

where b refers basic state. The Eq. (8) is solved for $\mathrm{S}_{\mathrm{b}}(\mathrm{z}, \mathrm{t})$ subject to the boundary condition Eq. (5), we get:

$$
\mathrm{S}_{b}(z, t)=\mathrm{S}_{b}(z)+\chi^{2} \delta_{s} \operatorname{Re}\left[\mathrm{S}_{1}(z, t)\right]
$$

\subsection{Perturbed State}

The finite amplitude perturbation quantities on the basic state are superposed in the form:

$$
\vec{q}=\vec{q}_{b}+\vec{q}^{\prime}, \rho=\rho_{b}+\rho^{\prime}, p=p_{b}+p^{\prime}, S=S_{b}+S^{\prime}
$$

Substituting Eq. (11) in Eqs. (1)-(4), and using the basic state solution, we get:

$\nabla \cdot \vec{q}^{\prime}=0$

$$
\frac{\partial \vec{q}^{\prime}}{\partial t}+2\left(\vec{\Omega} \times \vec{q}^{\prime}\right) \vec{k}=-\frac{1}{\rho_{0}} \nabla p+\alpha_{s} g S^{\prime}+\frac{v \vec{\mu}}{K} \overrightarrow{q^{\prime}}
$$

$\frac{\partial S^{\prime}}{\partial t}+w^{\prime} \frac{d S_{b}}{d z}+(\vec{q} \cdot \nabla) S^{\prime}=\kappa_{s} \nabla^{2} S^{\prime}+S_{i} S^{\prime}$,

In this problem only dimensional configuration is considered. So for two-dimensional convection, one can introduce the stream function as $\psi$ as $u^{\prime}=$ $\frac{\partial \psi}{\partial z}, w^{\prime}=-\frac{\partial \psi}{\partial x}$ Theses stream functions are assumed due to the continuum hypothesis given in Eq.(1). Using the dimensional analysis the physical variables are converted into unite less quantities. In this case the dimension less physical variables are defined by; $(x, y, z)=d\left(x^{*}, y^{*}, z^{*}\right), t=\frac{d^{2}}{\kappa_{s}} t^{*}, \vec{q}=\frac{\kappa_{s}}{d} \vec{q}^{*}$, $\psi=\kappa_{s} \psi^{*}, \quad S^{\prime}=\Delta S S^{*}$ and $\omega=\frac{\kappa_{S}}{d^{2}} \omega^{*}$, While eliminating the pressure term and dropping the asterisk, we obtain non-dimensional governing equations:

$$
\begin{aligned}
& \frac{1}{\operatorname{Vas}} \frac{\partial}{\partial t}\left(\nabla^{2} \psi\right)-\sqrt{T a} \frac{\partial V}{\partial z}=-R a_{S} \frac{\partial S}{\partial x}-\nabla^{2} \psi \\
& -\frac{\partial S_{b}}{\partial z} \frac{\partial \psi}{\partial x}-\left(\nabla^{2}+S_{i}\right) S=-\frac{\partial S}{\partial t}+\frac{\partial(\psi, S)}{\partial(x, z)}
\end{aligned}
$$

Also from the Eq. (13), the equation for $y$ component i.e $\mathrm{V}$ may be written as:

$\frac{1}{V a s} \frac{\partial V}{\partial t}+V=-\sqrt{T a} \frac{\partial \psi}{\partial z}$

The dimension less physical parameters in the above equations are given in nomenclature. The term sitting in Eq. (16) is the basic state solute $\mathrm{Sb}(\mathrm{z}, \mathrm{t})$ can be obtained from the Eq. (10) as:

$$
\begin{aligned}
& \frac{\partial S_{b}}{\partial z}=f_{1}(z)+\chi^{2} \delta_{S}\left[f_{2}(z, t)\right] \\
& f_{2}(z, t)=\operatorname{Re}\left[f(z) e^{(-i \Omega t)}\right] \\
& f_{1}(z)=-\frac{\sqrt{S_{i}}}{2 \sin \sqrt{S_{i}}}\left[\cos \sqrt{S_{i}}(1-z)+\cos \sqrt{S_{i}}(z)\right]
\end{aligned}
$$

$$
f(z)=A(m) e^{m z}+A(-m) e^{-m z}
$$

Since our study is restricted to slow variations in mass transfer the time may be re-scaled as $\tau=\chi^{2} \mathrm{t}$. In order to solve the above system of Eqs. (15)-(17) the following stress free and isothermal boundary 
conditions are considered.

$\psi=\frac{\partial^{2} \psi}{\partial z^{2}}=\frac{\partial V}{\partial z}=S=0$ at $\mathrm{z}=0$ and $\mathrm{z}=1$.

\section{FINITE AMPLITUDE EQUATION AND HEAT TRANSPORT}

The following asymptotic expansions for the physical variables are considered (given by Venezian (1969) and Malkus et al. (1958) to solve the Eqs.(1517)):

$$
\begin{aligned}
& \psi=\chi \psi_{1}+\chi^{2} \psi_{2}+\chi^{3} \psi_{3}+\ldots \\
& S=\chi S_{1}+\chi^{2} S_{2}+\chi^{3} S_{3}+\ldots \\
& V=\chi V_{1}+\chi^{2} V_{2}+\chi^{3} V_{3}+\ldots \\
& R a_{S}=R_{0 c}+\chi^{2} R_{2}+\chi^{4} R_{4}+\ldots
\end{aligned}
$$

where $\mathrm{R}_{0 c}$ is the critical solutal Rayleigh number at which the onset convection due to Buoyancy effect. After substitution of the above expansions into the Eqs. (15-17). The system of Eqs. (15-17) are solved for different orders of $\chi$. The reader may see the studies of Bhadauria et al. (2013a, 2014a-d, 2016, 2017, 2018 ).

\subsection{Lowest Order Case}

This order is just like linear problem, because no nonlinear term is appeared in this case. So the nonlinear effects are not counted in this state and treated as linear problem. The solutions of the lowest order system subject to the boundary conditions given in Eq. (22) are given by:

$$
\begin{aligned}
& \psi_{1}=A(\tau) \sin \left(k_{c} x\right) \sin (\pi z) \\
& S_{1}=\frac{-4 \pi^{2} k_{c} A(\tau)}{\beta_{1}^{2}\left(4 \pi^{2}-S_{i}\right)} \cos \left(k_{c} x\right) \sin (\pi z) \\
& V_{1}=-\pi \sqrt{T a} A(\tau) \sin \left(k_{c} x\right) \cos (\pi z)
\end{aligned}
$$

where $\beta=\beta^{2}-\mathrm{S}_{\mathrm{i}}$ and $\beta^{2}=k_{c}^{2}+\pi^{2}$. The critical value of the solutal Rayleigh number for the onset of stationary convection is given by:

$$
R_{0 c}=\frac{\beta_{1}^{2}\left(4 \pi^{2}-S_{i}\right)\left(\beta^{2}+\pi^{2} T a\right)}{4 \pi^{2} k_{c}^{2}}
$$

For the system without rotation $(\mathrm{Ta}=0)$ and internal solute $\left(S_{i}=0\right)$, we get

$$
\begin{aligned}
& R_{0 c}=\frac{\beta^{4}}{k_{c}^{2}}, \\
& \mathrm{k}_{\mathrm{c}}=\pi,
\end{aligned}
$$

which are the classical results of Lapwood (1948) and Bhadauria et al. (2013a). The oscillatory critical value of solutal Rayleigh number for the eigen value problem Eq.(15)-(17) with time-dependent periodic disturbances given is by Malashetty et al. (2008) Eq. 3.1 , it is obtained by:
$R_{0 c}^{o s c}=\frac{\beta^{2}\left(\operatorname{Vas} \beta_{1}^{2}-\omega^{2}\right)}{k_{c}^{2} \operatorname{Vas}}+\frac{\operatorname{Vas}^{2} \operatorname{Ta}\left(\operatorname{Vas} \beta_{1}^{2}-\omega^{2}\right)}{k c^{2}\left(\operatorname{Vas}^{2}-\omega^{2}\right)}$

and the growth rate $\omega$ is given by

$$
\omega^{2}=\operatorname{Vas}^{2}\left[\frac{\pi^{2} T a\left(V a s-\beta_{1}^{2}\right)}{\beta^{2}\left(\operatorname{Vas}-\beta_{1}^{2}\right)}-1\right]
$$

Setting $\omega=0$ stationary critical solutal Rayleigh number (given in Eq. (27) is obtained. The values of $R_{0 c}^{o s c}$ is calculated for only positive values of $\omega$ because the growth rate is positive. Also this critical oscillatory solutal Rayleigh number $R_{0 c}^{o s c}$ is a function of $\mathrm{Si}$ and $\mathrm{Ta}$. The readings of $\mathrm{R} 0 \mathrm{c}$, $\mathrm{kc}$ of stationary convection for different values of Ta and $\mathrm{Si}$ are given in Table 1.

Table 1. Readings of $R_{0 c}$, $k c$ for $T_{a}$ and $S_{i}$.

\begin{tabular}{|c|c|c|}
\hline $\mathrm{Ta}(\mathrm{Si}=1.0)$ & $\mathrm{R} 0 \mathrm{c}$ & $\mathrm{kc}$ \\
\hline 8 & 150 & 5.28987 \\
\hline 20 & 294 & 6.54796 \\
\hline 30 & 408 & 7.21758 \\
\hline 40 & 520 & 5.74011 \\
\hline $\mathrm{Si}=1.0(\mathrm{Ta}=8)$ & 150 & 5.28987 \\
\hline 2 & 141 & 5.14190 \\
\hline 3 & 134 & 4.97014 \\
\hline 4 & 126 & 4.77846 \\
\hline
\end{tabular}

\subsection{Second Order Case}

In this order the nonlinear effects enter to the system through Jacobian term of the Eq. (16). At this order the following relations are obtained:

$\psi_{2}=0$

$-\left(\nabla^{2}-S_{i}\right) S_{2}=\frac{\partial \psi_{1}}{\partial x} \frac{\partial S_{1}}{\partial z}-\frac{\partial \psi_{1}}{\partial z} \frac{\partial S_{1}}{\partial x}$

$V_{2}=0$

The second order solutions subject to the boundary conditions Eq. (22) are given by:

$\psi_{2}=0$,

$S_{2}=-\frac{2 \pi^{3} k_{c}^{2} A^{2}(\tau)}{\beta_{1}^{2}\left(4 \pi^{2}-S_{i}\right)^{2}} \sin (2 \pi z)$

$V_{2}=0$

The reader may not that the second order system solutions are basically depends on the first order system solutions. This is the reason we call the stability as weakly nonlinear stability analysis. Here the critical solutal Rayleigh number is assumed to be near to threshold of onset convection. The horizontal averaged Sherwood number $\operatorname{Sh}(\tau)$ for stationary mode of convection is given by: 


$$
\operatorname{Sh}(\tau)=1+\frac{\left[\frac{k_{c}}{2 \pi} \int_{0}^{\frac{2 \pi}{k_{c}}}\left(\frac{\partial S_{c}}{\partial z}\right) d x\right]_{z=0}}{\left[\frac{k_{c}}{2 \pi} \int_{0}^{\frac{2 \pi}{k_{c}}}\left(\frac{\partial S_{b}}{\partial z}\right) d x\right]_{z=0}}
$$

Substituting the expression of S2 and $\left(\partial \mathrm{S} \_b\right) / \partial z i n$ Eq. (34) and simplifying we get:

$$
\operatorname{Sh}(\tau)=1+\frac{4 \pi^{2} k_{c}^{2} \sin \sqrt{S_{i}}}{\beta_{1}^{2}\left(4 \pi^{2}-S_{i}\right)^{2} \sqrt{S_{i}} \cos \sqrt{S_{i}}} A^{2}(\tau)
$$

This Sherwood number is to measure the mass transfer in a system as a function of finite amplitude of convection.

\subsection{Third Order Case}

At this stage the following system is obtained involving many terms in RHS:

$\left[\begin{array}{ccc}\nabla^{2} & R_{0 c} \frac{\partial}{\partial x} & -\sqrt{T a} \frac{\partial}{\partial z} \\ -\frac{\partial S_{b}}{\partial z} \frac{\partial}{\partial x} & -\left(\nabla^{2}+S_{i}\right) & 0 \\ \sqrt{T a} \frac{\partial}{\partial z} & 0 & 1\end{array}\right]\left[\begin{array}{l}\psi_{3} \\ S_{3} \\ \nabla_{3}\end{array}\right]=\left[\begin{array}{l}R_{31} \\ R_{32} \\ R_{33}\end{array}\right]$

where

$R_{31}=-\frac{1}{V a s} \frac{\partial}{\partial \tau}\left(\nabla^{2} \psi_{1}\right)-R_{2} \frac{\partial S_{1}}{\partial x}$

$R_{32}=-\frac{\partial S_{1}}{\partial \tau}+\frac{\partial \psi_{1}}{\partial x} \frac{d S_{2}}{d z}+\delta_{2} f_{2} \frac{\partial \psi_{1}}{\partial x}$

$R_{33}=-\frac{1}{\text { Vas }} \frac{\partial V_{1}}{\partial \tau}$

Substituting the first and second order solutions into the Eqs. (37)-(39), the expressions for R31, R32 and R33 will be simplified. In general, finding the solutions at this state is difficult due to nonlinearity. The following solvability condition is used to derive finite amplitude equation.

$\int_{0}^{1} \int_{0}^{\frac{2 \pi}{k_{c}}}\left(R_{31} \hat{\psi}_{1}+R_{32} \hat{S}_{1}+R_{33} \hat{V_{1}}\right) d x d z=0$

the Ginzburg-Landau equation (GLE) is obtained for the existence of the solution at third order for stationary mode of convection:

$Q_{1} A^{\prime}(\tau)+F(\tau) A(\tau)-Q_{2} A(\tau)^{3}=0$

where $\hat{\psi}_{1}, \hat{S}_{1}$ and $\widehat{V}_{1}$ are the adjoint solutions of first order system,

$Q_{1}=\left[\frac{\beta^{2}-T a \pi^{2}}{\operatorname{VaS}}+\frac{4 R_{0 c} \pi^{2} k_{c}^{2}}{\beta_{1}^{2}\left(4 \pi^{2}-S_{i}\right)}\right]$

$F(\tau)=\left[\frac{4 R_{2} \pi^{2} k_{c}^{2}}{\beta_{1}^{2}\left(4 \pi^{2}-S_{i}\right)}-\delta_{S} R_{0 c} I_{1},\right]$
$Q_{2}=\left[\frac{4 R_{0 c} \pi^{4} k_{c}^{4}}{\beta_{1}^{4}\left(4 \pi^{2}-S_{i}\right)^{2}}\right]$,

$I_{1}=\int_{0}^{1} f_{2}(z, s) \sin ^{2}(\pi z) d z$

The GL Eq. (41) is also known as Bernoulli equation. But finding its analytical solution is difficult, due to its non-autonomous nature. So it has been solved numerically using the in-built function ND-Solve of Mathematica 8 , subject to the suitable initial amplitude $\mathrm{A}(0)=\mathrm{a}$. In our calculations we may use $\mathrm{R} 2=\mathrm{R} 0 \mathrm{c}$, to keep the parameters up to minimum. The Sherwood number $\operatorname{Sh}(\tau)$ given in Eq. (36) is numerically evaluated by obtaining the finite amplitude $\mathrm{A}(\tau)$ from the Eq. (41). The Eq. (41) is a non-autonomous Bernoulli equation.

\subsection{Amplitude for Unmodulated Case}

In the case of unmodulated porous layer the above Ginzburg-Landau equation (GLE) can be written as

$Q_{1} A_{u}^{\prime}(\tau)+F(\tau) A_{u}(\tau)-Q_{2} A_{u}(\tau)^{3}=0$

where $\mathrm{A}_{\mathrm{u}}(\tau)$ is an amplitude of convection for unmodulated case. The coefficients $\mathrm{Q}_{1}$ and $\mathrm{Q}_{2}$ have the same expressions as given in the Eq. (41). The term $\mathrm{F}(\tau)$ is given by $F=\frac{4 R_{2} \pi^{2} k_{c}^{2}}{\beta_{1}^{2}\left(4 \pi^{2}-S_{i}\right)}$. The solution of Eq. (42) is given by

$$
A_{u}(\tau)=\frac{1}{\sqrt{\left(\frac{Q_{2}}{2 F}\right)+C_{1} \operatorname{Exp}\left[\frac{2 F}{Q_{1}}\right]}}
$$

where $C_{1}$ is a parameter, it can be calculated for given suitable initial condition. The horizontal averaged Sherwood number in this case is obtained from Eq. (36) by using the value of $\mathrm{A}_{\mathrm{u}}(\tau)$ in place of $\mathrm{A}(\tau)$.

\section{RESULTS AND DISCUSSION}

This paper presents a weakly nonlinear stability analysis to investigate the effect of solutal modulation and internal solutal on mass transport. The system of nonlinear equations are solved using the analysis of Ginzburg-Landau model (see Bhadauria et al. (2012, 2013a, 2014a-d, 2015, 2016). This GL equation gives finite amplitude of convection as a function of time. Due to its nonautonomous nature the unmodulated system is solved analytically and modulated system numerically. The solutal modulation of Darcy convection has been assumed to be of third order only. Which shows that only small amplitude of solute modulation is considered. Before writing the discussion of the results, we mention some features of the following aspects of the problem. In order to analyze the effect of solutal modulation, three types 
of modulations are taken: (i) in-phase modulation (IPM) $(\theta=0)$,(ii) out-phase modulation (OPM) $(\theta=$ $\pi$ ), and (iii) lower boundary modulations (LBMO) $(\theta$ $=-\mathrm{i} \infty)$. At first the results related to OPM case will be presented. The results corresponding to IPM and LBMO will not be presented due to repetition of the figures but they are compared. In OPM case both the plates are modulated differently. In IPM case both the plates are modulated in the same manner. The reader may observe easily the difference of different types of modulations on Darcy convection through the nature of the figures.

A weakly nonlinear analysis is performed to obtain mass transport in a porous layer. This finite amplitude analysis is obtained based on linear stability analysis. The external solutal modulation is important to control mass transfer in a porous media. Therefore, this paper is constructed to find mass transfer in the system. The values of the parameters are taken within their range where one can obtain the results clearly. Further the values of $S_{i}$ are considered very small so that it will not affect the effect of modulation on the system. In this paper the values of $\mathrm{S}_{\mathrm{i}}$ are taken from 0.5 to 8. Also the values of Vadasz number and Taylor number are consider within the range of the solutions. The amplitude of modulation $\delta_{\mathrm{s}}$ is consider between 0.1 to 0.3 and frequency of modulation between 2 to 50 . The main results are presented in the next paragraph (given in figures from Fig. 2 to Fig. 7).

In Fig. 2 the effect of solute Rayleigh number on the marginal stability curves for the Taylor number Ta and internal solutal number Si is depicted. The effect of Taylor number, Ta on the onset of convection when $\mathrm{Si}$ is fixed is given in Fig. 2a. It is clear that the minimum of Rayleigh number for stationary convection increases with the Taylor number, indicating that the effect of rotation is to enhance the stability. Thus higher rotational rates of the porous layer stabilizes the system and may reduce momentum transfer in the system. In Fig. $2 b$ the effect of internal solutal number $\mathrm{Si}$ is presented on the marginal stability curves. It is observed that with increase of the value of Si the minimum of stationary solutal Rayleigh number decreases. Therefore, the effect of $\mathrm{Si}$ is to advance the onset of stationary convection. Thus for moderate values of $\mathrm{Si}$ the system may attain stable mode, but for higher values it may attains unstable mode. The effect of $\mathrm{Ta}$ and $\mathrm{Si}$ on R0c is drawn in Fig. 2c. It is evident that Ta promotes stabile convection and $\mathrm{Si}$ promotes unstable convection. In Fig. 2d, it is quite natural to see that the oscillatory convection sets in before stationary convection. The internal solutal Rayleigh number having destabilizing effect and rotation Ta having stabilizing effect on the system. The regions for stationary and oscillatory convection is presented in this figures. It is concluded that the effect of $\mathrm{Ta}, \mathrm{Si}$ and Vas on both R0c and R0c ${ }^{\text {osc }}$ shows the same stabilize and destabilize effect on the system.

The study of mass transfer is presented in the Figs.
3-5. These figures are drawn based on Sherwood number Sh versus slow time $\tau$ for the case of solute modulation. From the figures it is found that for small values of time $\tau$, the value of $\operatorname{Sh}(\tau)$ does not alter and remains almost constant. When the time is increasing then Sh increasing and finally becomes

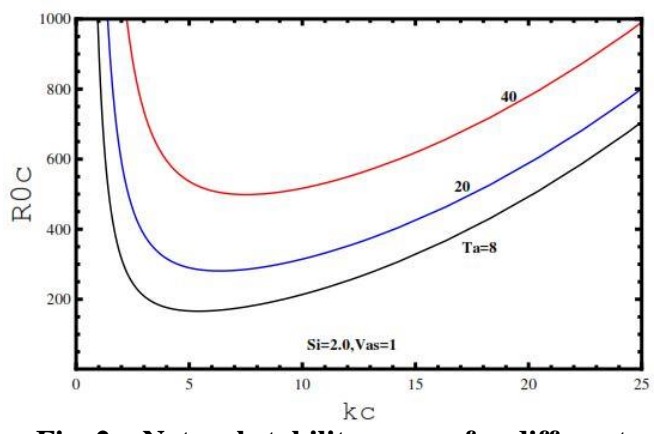

Fig. 2a. Natural stability curves for different values of Ta.

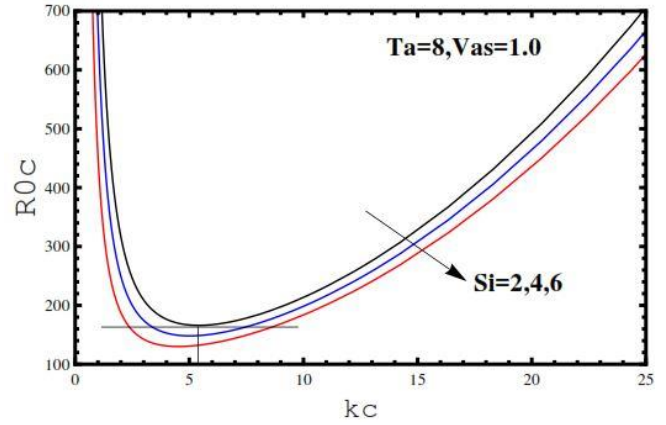

Fig. 2b. Natural stability curves for different values of $\mathrm{Si}$.

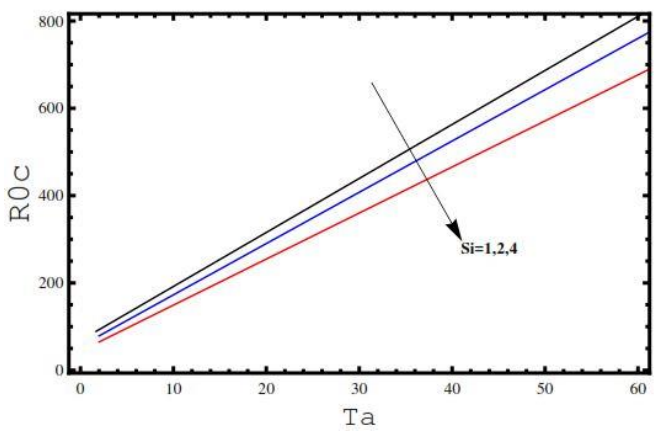

Fig. 2c. Variation of critical Rayleigh number Roc with different values of $\mathrm{Si}$.

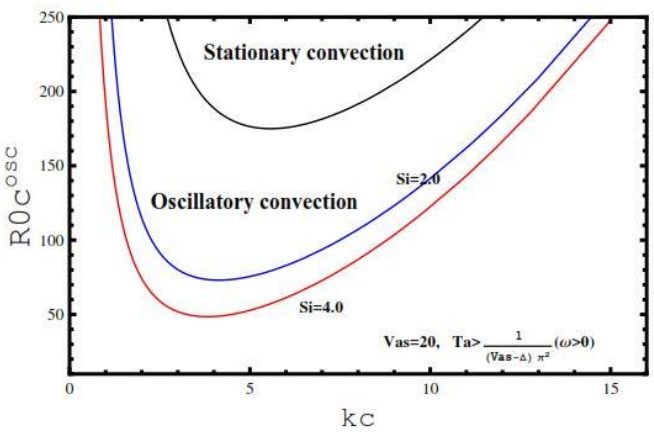

Fig. 2d. Variation of critical Rayleigh oscillatory number Roc with different values of $\mathrm{Si}$. 

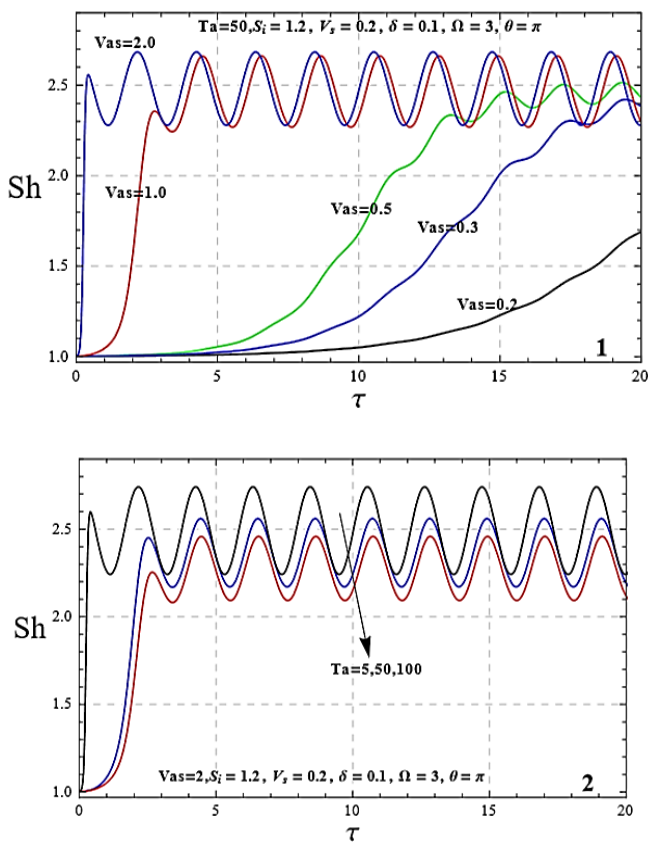

Fig. 3. The effect of Vas Ta on Sh versus T.

oscillatory on further increasing $\tau$. This oscillatory nature is due to time periodic solting at the boundaries. It is clear from the figures that $\operatorname{Sh}(\tau)$ starts with 1 showing the conduction state. Further increment in Sh shows convective state in the system.

The mass transport results: Fig. 3(1) presents results corresponding to Vas (solute Vadasz number) on Sherwood number Sh. According to Vadasz (1998) the values of Vas are considered around 1. Vadasz pointed that there are some porous applications such as mushy layer in solidification of binary alloys and fractured porous medium the value of Vas may be considered to be unity order. Therefore, the quotient of local time derivative term of Vas in the present study has been retained. It is clear from the Fig. 3(1) that there is appreciable enhancement in mass transport on increasing Vas, thereby advancing the onset of convection. The similar effect of Vas may observe in the following studies of Bhadauria et al. (2013a, 2014a,d) and Srivastava et al. (2013).

The effect of rotation (Ta) presented in the Fig. 3(2). It is natural phenomenon to see that rotation reduces mass transfer in the medium due to the presence of centrifugal force acting opposite to the motion of the fluid. Thus reduces mass transfer and confirms the results of Desaive et a. (2002), for thermal and gravity modulation Malashetty et al.(2008), Kiran et al.(2017, 2018c; 2016a,b; 2020) and for solutal modulation Gupta et al. (2017), Gupta (2018) and Praksh et al.(2018). The effect of internal solute number $\mathrm{Si}$ is presented in the Figs. 3(1) and 3(2). The positive values of $\mathrm{Si}$ represents source of mass and enhances mass transfer in the medium (in Fig. 4(1)). But for negative values of $\mathrm{Si}$ there is drastic reduction in mass transfer see Fig. 4(2). Thus, the duel nature of internal solute number $\mathrm{Si}$ is observed.

The effect of solute modulation in terms of $\left(\Omega_{\mathrm{f}}, \delta_{\mathrm{s}}\right)$ is presented in the Figs. 4(3), 4(4). In Fig. 4(3), we observe that an increment in $\Omega_{f}$ decreases the magnitude of Sh, and shortens the wave-length of oscillations. As the frequency of modulation increases from 1 to 30 , the magnitude of $\mathrm{Sh}$ decreases, and so is the modulation effect. When the value of $\Omega_{\mathrm{f}}$ is increased further, we find that at $\Omega_{\mathrm{f}}=$ 30 , the effect of modulation on solutal instability disappears altogether.

$S h_{\Omega}=30<S h_{\Omega}=10<S h_{\Omega}=4<S h_{\Omega}=1$
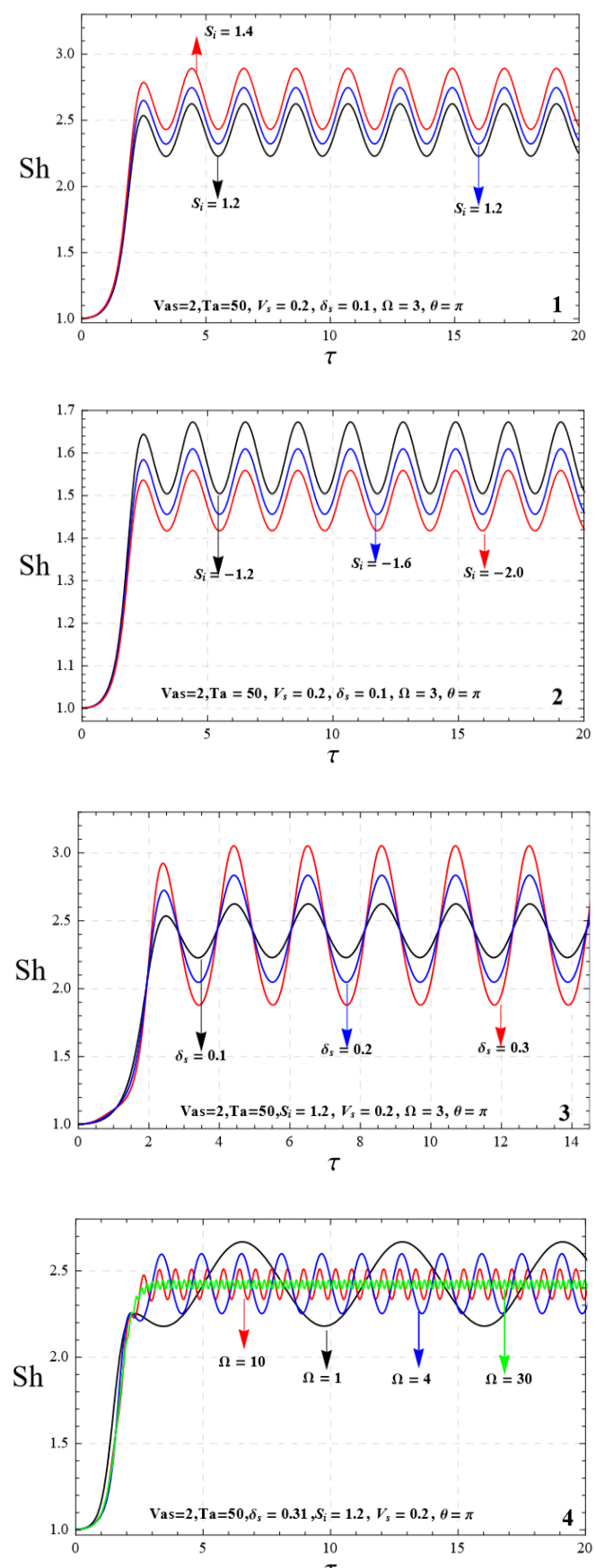

Fig. 4. The effect of Vas $S_{i} \delta_{s}$ and $\omega$ on Sh versus $\tau$.

Further, it is found in the Fig. 4(4) that the effect of 
amplitude of modulation is to enhance the magnitude of Sh, thus increasing the mass transport. We note that the following is due to the influence of amplitude on mass transport.

$$
S h_{\delta=0.1}<S h_{\delta=0.2}<S h_{\delta=0.3}
$$

These results confirms the results of Gupta et al. (2017), Gupta (2018) and Praksh et al. (2018) for solutal modulation and for thermal and gravity modulations Bhadauria et al.(2007, 2008, 2013a, 2014a-d, 2018). It is found that the magnitude of Sh for LBMO is greater than that obtained in case of IPM, but less than that of OPM as shown below, the results are given in Fig. 5(1):

$S h_{I P M}<S h_{I . B M O}<S h_{O P M}$
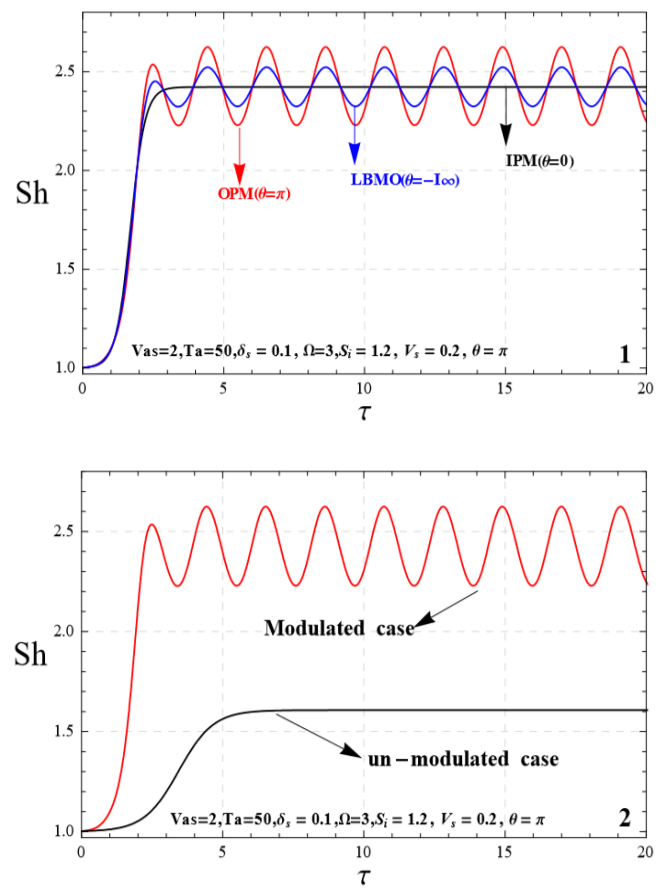

Fig. 5. Different profiles comparision.

The above equation tells that OPM case is the better option to enhance the mass transfer in a porous medium. The reader may refer the article by Gupta et al. (2017) and Gupta (2018). The comparison of modulated and un-modulated case is given in Fig. 5(2). Finite amplitude is obtained analytically using Eq.43 for un-modulated case. It is found that modulated system transfer more mass transfer than un-modulated system. The reason is due to the concentration heavy at the upper plate than lower plate.

While solving the Eqs. $(24,25)$ the streamlines and isohaline are drawn in Figs. 6 and 7. These streamlines and isohalines are depicted for rotation speed modulation at various instances $\tau=$ $0.0,0.1,0.3,0.5,1.0$ and 2.0 for $\operatorname{Vas}=1.0, \mathrm{~S}_{i}=1.0, \mathrm{Ta}$ $=50, \delta_{s}=0.1$ and $\Omega_{f}=3.0$. From the Figs. $6 \mathrm{a}$, b, it is found that initially when the time is small the magnitude of streamlines is also small. At initial state
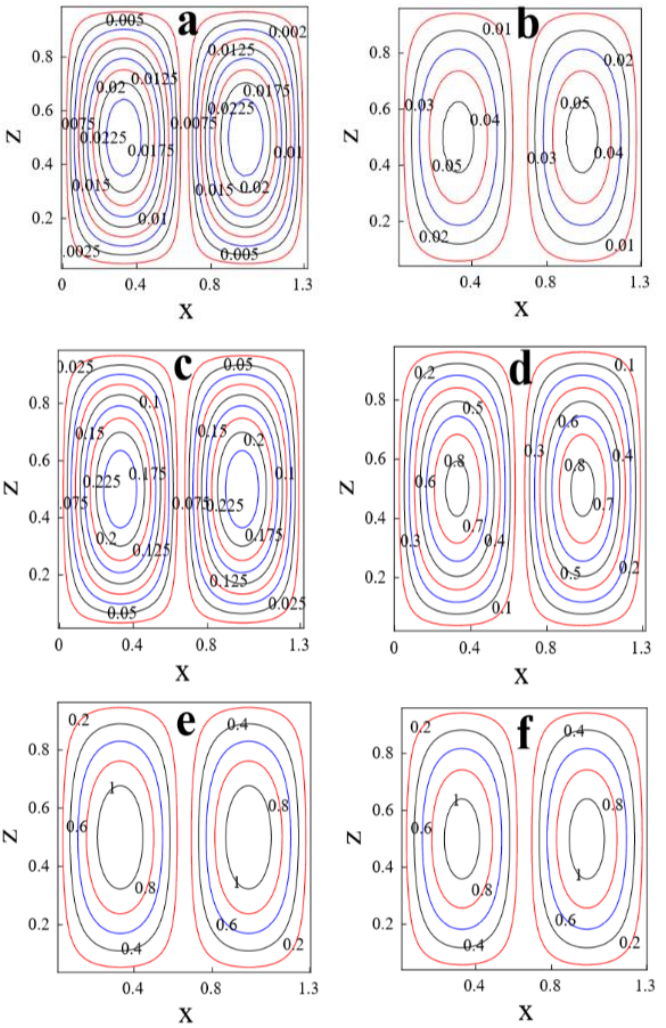

Fig.6. Streamlines for $\tau$ (a) $0.0($ b) $0.1($ c) $0.3(d)$ $0.5(\mathrm{e}) 1.0(\mathrm{f}) 2.0$
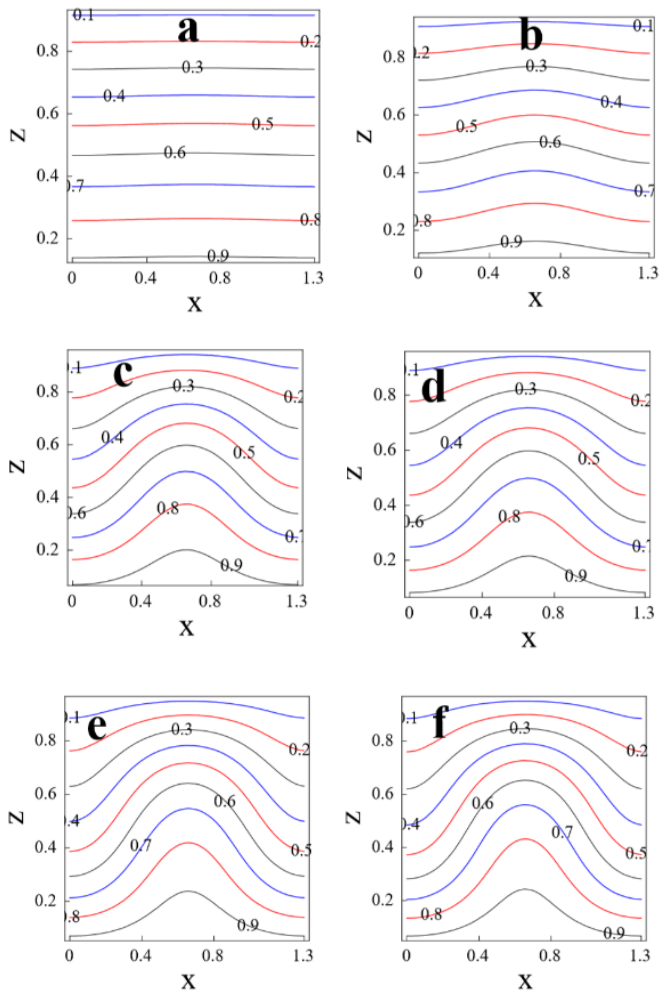

Fig.7. Isotherms for $\tau$ (a) $0.0($ b) $0.1($ c) $0.3(d)$ $0.5(\mathrm{e}) 1.0(f) 2.0$ 
even the isohaline $7 \mathrm{a}, \mathrm{b}$ are straight lines showing the system is in conduction state. Further as time passes the magnitude of streamlines increases and the isohaline loses their evenness. This shows that the convection is in progress. Convection becomes faster on further increasing the value of time $\tau$. However, the system achieves its steady state beyond the time $\tau=1.0$ as there is no change in the streamlines and isohaline $6 \mathrm{e}, \mathrm{f}-7 \mathrm{e}, \mathrm{f}$.

\section{CONCLUSIONS}

The combined effect of internal solute and solutal modulation on convection in a porous layer, has been investigated while employing a weakly non-linear analysis. Using the Ginzburg-Landau equation (GLE) finite amplitude is obtained. The results have been presented in terms of the Sherwood number, and the effect of various parameters depicted graphically. The following observations are made.

1. The stability curves are shoing that oscillatory convection sets in before stationary convection.

2. The effect of $S_{i}>0$ case is to increase and $\mathrm{Sh}$ $<0$ case decrease the mass transfer.

3. The effect of Taylor number is to reduce the mass transfer.

4. The effect of amplitude $\delta_{\text {s }}$ of modulation is to enhances mass transfer.

5. The effect of frequency $\Omega_{\mathrm{f}}$ of modulation is to diminish the mass transfer.

6. The effect of modulation can be seen only for OPM and LBMO cases and obtained better mass transfer in the medium.

7. The modulated system is efficient than unmodulated system.

8. The un-evenness of streamlines and isotherms are showing convective motions.

9. Streamlines and isotherms loses their evenness as time passes showing the flow of mass transport through conduction to convection.

\section{ACKNOWLEDGEMENT}

The author Dr. Palle Kiran is grateful to his Institute CBIT for providing research specialities in the Department. He also thanks Smt. D. Sandhya Shree (Board member of CBIT) for her encouragement towards the research. He also thanks the HOD Prof. Raja Reddy, Department of Mathematics, CBIT for his support and encouragement. Finally, the author PK is grateful to the referees for their most valuable comments that improved the paper considerably.

\section{REFERENCES}

Bhadauria, B. S. (2007). Double diffusive convection in a rotating porous layer with modulated temperature on the boundaries. Journal of Porous Media 10(6), 569-584.

Bhadauria, B. S. (2008). Effect of temperature modulation on Darcy convection in a rotating porous medium. Journal of Porous Media 11(4), 361-375.

Bhadauria, B. S., P. G. Siddheshwar, J. Kumar and P. Om (2012). Suthar. Weakly Nonlinear Stability Analysis of Temperature/GravityModulated Stationary Rayleigh-Bénard Convection in a Rotating Porous Medium. Transport in Porous Media 92, 633-647.

Bhadauria, B. S. and P. Kiran (2013a). Heat transport in an anisotropic Porous medium saturated with variable viscosity liquid under temperature modulation. Transp Porous Media 100, 279295.

Bhadauria, B. S. and P. Kiran (2014a). Weakly nonlinear oscillatory convection in a viscoelastic fluid saturating porous medium under temperature modulation. International Journal of Heat and Mass Transfer 77, 843-851.

Bhadauria, B. S. and P. Kiran (2014b). Heat and mass transfer for oscillatory convection in a binary viscoelastic fluid layer subjected to temperature modulation at the boundaries. International Communications in Heat and Mass Transfer 58, 166-175.

Bhadauria, B. S. and P. Kiran (2014c). Weak nonlinear oscillatory convection in a viscoelastic fluid layer under gravity modulation. International Journal of NonLinear Mechanics 65, 133-140.

Bhadauria, B. S. and P. Kiran (2014d). Weak nonlinear oscillatory convection in a viscoelastic fluid saturated porous medium under gravity modulation. Transport in Porous Media 104, 451-467.

Bhadauria, B. S. and P. Kiran (2016). Weakly nonlinear oscillatory convection in a rotating fluid layer under temperature modulation. The ASME Journal of Heat Transfer 138, 051702(1$10)$.

Bhadauria, B. S. and P. Kiran (2014). Effect of rotational speed modulation on heat transport in a fluid layer with temperature dependent viscosity and internal heatsource. Ain Shams Engineering Journal 5, 1287-1297.

Bhadauria, B. S. and P. Kiran (2015). Weak nonlinear double diffusive magneto-convection in a newtonian liquid under gravity modulation. Journal of Applied Fluid Mechanics 8(4) 735-746

Bhadauria, B. S. and V. Kumar (2018). Convective Motion in Nanofluid Under Variable Rotational 
Speed. Advanced Science, Engineering and Medicine 10, 712-718.

Desaive, T., M. Hennenberg. and G. Lebon (2002). Thermal instability of a rotating saturated porous medium heated from below and submitted to rotation. The European Physical Journal B. 29, 641-647.

Gresho, P. M. and R. L. Sani (1970). The effects of gravity modulation on the stability of a heated fluid layer. Journal of Fluid Mechanics 40, 783-806.

Gupta, V. K., A. Kumar and A. K. Singh (2017). Analytical study of weakly non-linear mass transfer in rotating fluid layer under timeperiodic concentration/gravity modulation. International Journal of Non-Linear Mechanics 97, 22-29.

Gupta, V. K. (2018). Study of mass transport in rotating couple stress liquid under concentration modulation. Chinese Journal of Physics 56, 911-921.

Horton, C. W. and F. T. Rogers (1945). Convection currents in a porous medium. Journal of Applied Physics 16, 367-370.

Ingham, D. B. and I. Pop (2005). Transport Phenomena in Porous Media. Vol. III, Elsevier, Oxford.

Kiran, P. and Y. Narasimhulu (2017). Centrifugally driven convection in a nanofluid saturated rotating porous medium with modulation. Journal of Nanofluids 6(3), 513-523..

Kiran, P., S. H. Manjula and Y. Narasimhulu (2018a). Weakly nonlinear oscillatory convection in a viscoelastic fluid saturated porous medium with throughflow and temperature modulation. International Journal of Applied Mechanics and Engineering 23, 01-28.

Kiran, P. and Y. Narasimhulu (2018b). Internal heating and thermal modulation effects on chaotic convection in a porous medium. Journal of Nanofluids 7(3), 544-555.

Kiran, P., S. H. Manjula and Y. Narasimhulu (2018c). Oscillatory convection in a rotating fluid layer under gravity modulation. Journal of Emerging Technologies and Innovative Research 5(8), 227-242.

Kiran, P. and B. S. Bhadauria (2017a). Weak nonlinear rotating Bénard convection with modulation using Ginzburg- Landau model. Int Journal of Science, Technology and Society 3(1) 48-57.

Kiran, P., Bhadauria, B. S. and Y Narasimhulu (2017b). Nonlinear throughflow effects on thermally modulated rotating porous medium. Journal of Applied Nonlinear Dynamics 6, 27-44.
Kiran, P., B. S. Bhadauria and Y. Narasimhulu (2017c). Oscillatory magneto- convection under magnetic field modulation. Alexandria Engineering Journal 57, 445-453.

Kiran, P. and B. S. Bhadauria (2016a). Throughflow and rotational effects on oscillatory convection with modulated. Nonlinear Studies 23(3), 439-455.

Kiran, P. and B. S. Bhadauria (2016b). Weakly nonlinear oscillatory convection in a rotating fluid layer under temperature modulation. ASME Journal of Heat Transf. 138, 051702.

Kiran, P. (2016c). Throughflow and Gravity Modulation Effects on Heat Transport in a Porous Medium. Journal of Applied Fluid Mechanics 9 (3), 1105-1113.

Kiran, P. and S. H. Manjula (2020). The complex Ginzburg Landau model for an oscillatory convection in a rotating fluid layer. International Journal of Applied Mechanics and Engineering 25, 75-91.

Kiran, P. (2016d). Nonlinear throughflow and internal heating effects on vibrating porous medium. Alexandria Engineering Journal 55, 757-767.

Lapwood, E. R. (1948). Convection of a fluid in a porous medium. Proceedings of the Cambridge Philosophical Society 44, 508-521.

Mohammed, S. I. (2013). Radiation Effects on Mass Transfer Flow through a Highly Porous Medium with Heat Generation and Chemical Reaction. ISRN Computational Mathematics 2013, 01-09.

Malashetty, M. S. and H. Rajashekhar (2008). Linear and non-linear double diffusive convection in a rotating porous layer using a thermal nonequilibrium model. JInt Journal of Non-Linear Mechanics 43, 600-621.

Malashetty, M. S. and M. Swamy (2007). Combined effect of thermal modulation and rotation on the onset of stationary convection in porous layer. Transport in Porous Media 69, 313-330.

Malkus, W. V. R. and G. Veronis (1958). Finite amplitude cellular convection. Journal of Fluid Mechanics 4, 225.

Moli Z, Z. Qiangyong and S. Wang (2014). Linear and nonlinear stability analysis ofdouble diffusive convection in a maxwell fluid saturated porous layer with internal heat source. Journal of Applied Mathematics 2014, 01-12.

Nield, D. A. and A. Bejan (2006). Convection in Porous Media. 3rd edn. Springer-Verlag, New 
York.

Patil, P. R., C. P. Parvathy and K. S. Venkatakrishnan (1990). Effect of rotation on the stability of a doubly diffusive layer in a porous medium. International Journal of Heat and Mass Transfer 33, 1073-1080.

Pearlstein, A. J. (1981). Effect of rotation on the stability of a doubly diffusive fluid layer. Journal of Fluid Mechanics 103, 389-412.

Praksh, K., A. Kumar and V. K. Gupta (2018). Magento-solutal convection in Newtonian fluid layer with solutal modulated boundaries. Internaional Journal of Nonlinear Mechanics 107, 86-93.

Raghunatha, K. R. and I. S. Shivakumara (2019). Double-diffusive convection in an oldroyd-b fluid layer-stability of bifurcating equilibrium solutions. Journal of Applied Fluid Mechanics 12, 85-94.

Singh, A. K. and A. K. Singh (2019). Influence of heat and mass transfer on free convection of micropolar fluid between vertical concentric cylinders. Journal of Applied Fluid Mechanics 12(5), 1539-1545.

Srivastava A., B. S. Bhadauria, P. G. Siddheshwar and I. Hashim (2013). Heat transport in an anisotropic porous medium saturated with variable viscosity liquid under g-jitter and internal heating effects. Transport in Porous Media 99, 359-376.

Srivastava, A., B. S. Bhadauria and A. K. Singh (2018). Study of heat and mass transport in Bénard-Darcy convection with G-Jitter and variable viscosity liquids in a porous layer with internal heat source. Journal of Applied Fluid Mechanics 11(5), 1217-1229.

Om, S. P., B. S. Bhadauria and A. Khan (2011). Rotating Brinkman-Lapwood Convection with Modulation. Transport in Porous Media 88, 369-383.

Om, S. P., B. S. Bhadauria and A. Khan (2009). Modulated centrifugal convection in a rotating vertical porous layer distant from the axis of rotation. Transport in Porous Media 79(2), 255-264.

Umavathi, J. C. (2013). Effect of thermal modulation on the onset of convection in a porous medium layer saturated by a nanofluid. Transport in Porous Media 98, 59-79.

Vadasz, P. (1998). Coriolis effect on gravity-driven convection in a rotating porous layer heated from below. Journal of Fluid Mechanics 376, 351-375.

Vafai, K. (2005). Handbook of Porous Media. Taylor and Francis/CRC, Lon-don/Boca Raton.

Venezian, G. (1969). Effect of modulation on the onset of thermal convection. Journal of Fluid Mechanics 35, 243-254.

Yadav, D. (2018) The influence of pulsating throughflow on the onset of electro-thermoconvection in a horizontal porous medium saturated by a dielectric nanofluid. Journal of Applied Fluid Mechanics 11(6), 1679-1689. 